\title{
Tratamento Cirúrgico do Câncer da Porção Distal do Têrço Inferior do Reto pela Ressecção Anterior Ultrabaixa e Interesfinctérica com Anastomose Coloanal por Videolaparoscopia
}

\author{
Surgical Treatment of Ultra-low Rectal Cancer by Laparoscopic Coloanal \\ Anastomosis and Intersphincteric Resection
}

\author{
JOSÉ REINAN RAMOS, TSBCP 1,2,3,4,5,6; RONALDO MACHADO MESQUITA, TSBCP ${ }^{1,2}$; \\ EDUARDOA. VALORY, ASBCP ${ }^{1,2}$; FELIPE SANTOS, ASBCP ${ }^{1,2}$
}

\author{
${ }^{1}$ Membro Titular do SBCP; ${ }^{2}$ Membro Titular do CBC; ${ }^{3}$ Membro Titular da Academia de Medicina do \\ Rio de Janeiro; ${ }^{4}$ Mestre em Cirurgia pela Universidade Illinois-USA, ${ }^{5}$ Certificação em Videocirurgia pela AMB; \\ ${ }^{6}$ Ex-Presidente do Colégio Brasileiro de Cirurgiões.
}

\begin{abstract}
RAMOS JR; VALORY EA; SANTOS F; MESQUITA RM. Tratamento Cirúrgico do Câncer da Porção Distal do Têrço Inferior do Reto pela Ressecção Anterior Ultrabaixa e Interesfinctérica com Anastomose Coloanal por Videolaparoscopia. Rev bras Coloproct, 2009;29(3): 314-324.
\end{abstract}

Resumo: Objetivo: Identificar as complicações pós-operatórias, a duração da internação hospitalar, os resultados funcionais clínicos, e os resultados oncológicos num seguimento médio de 2,5 anos nos pacientes com câncer do reto distal submetidos à ressecção anterior ultrabaixa e interesfinctérica com anastomose coloanal por videolaparoscopia. Casuística e Métodos: De um total de 491 pacientes operados pelo acesso videolaparoscópico, foram selecionados para esse estudo prospectivo 13 doentes, nove do sexo feminino,com câncer da porção distal do reto inferior entre os 172 pacientes com câncer do reto.Nenhum tumor T4 ou com resposta completa à quimiorradioterapia foi selecionado. A quimiorradioterapia neo-adjuvante foi aplicada em 8 doentes. Resultados: A taxa de complicaçãos pós-operatória foi de $23,1 \%$, sendo de $7,7 \%$ o índice de fístula anastomótica. A mortalide foi nula. A alta hospitalar ocorreu até $07^{\circ}$ dia de pós-operatório para 8 pacientes $(61,5 \%), 4$ dos quais no quinto dia.. 0 número médio de linfonodos por peça foi 13 . A margem distal média foi de $1,5 \mathrm{~cm}$. A margem circunferencial foi positiva em um caso (7,7\%). Evacuação fracionada foi relatada por 11 pacientes $(91 \%)$ e incontinência fecal por 5 pacientes $(41 \%)$. Onze pacientes $(84 \%)$ estão satisfeitos com a operação. Um paciente continua ileostomizado (7,7\%). Em um seguimento médio de 30 meses ocorreu uma recidiva local $(\mathbf{7 , 7 \%})$ e dois casos de metástase pulmonar $(\mathbf{1 5 , 4} \%)$. As três pacientes faleceram da doença. Dez pacientes $(\mathbf{7 7 \%})$ estão sem doença. CONCLUSÕES: Apesar da pequena casuística, a analise permitiu as seguintes conclusões: a) A técnica empregada mostrou ser viável e segura, pois apresentou baixo índice de complicação pós-operatória e mortalidade nula; b) $O$ emprego dessa técnica permitiu período de internação hospitalar pós-operatório curto; c) Apesar dos resultados funcionais com avaliação clínica regular, evitou-se a colostomia definitiva em $92,3 \%$ dos doentes; d) A utilização dessa técnica não comprometeu os resultados oncológicos num período médio de 30 meses de seguimento.

Discritores: Ressecção Interesfictérica, Câncer do Reto, Laparoscopia.

\section{INTRODUÇÃO}

A ressecção abdominoperineal do reto idealizada por E. Miles (1) em 1908 foi considerada a operação "padrão ouro" do século passado para o tratamento do adenocarcinoma do reto. O estabelecimento de critérios técnicos, sem prejuízo da radicalidade, tornou as operações com preservação esfincteriana, associada ou não a tratamentos neo-adjuvantes, as mais realizadas atualmente, com baixos índices de complicação pós-operatória e de recidiva local, sem a necesidade de colostomia definitiva. A ressecção ante- 
Tratamento Cirúrgico do Câncer da Porção Distal do Têrço Inferior do Reto pela Ressecção Anterior Ultrabaixa e Interesfinctérica com Anastomose Coloanal por Videolaparoscopia José Reinan Ramos e Cols. rior do reto baixa ou ultra-baixa com excisão total do mesorreto e preservação dos nervos pélvicos, seguida de anastomose colo-anal com bolsa colônica em $\mathbf{J}$ por duplo grampeamento, é aceita como a operaçao "padrão ouro" desse início de milênio, para o tratamento do câncer do reto distal.

Com o aumento dos conhecimentos sobre a disseminação do câncer do reto e melhor avaliação da importância não só da margem de ressecção distal (2 $\mathrm{cm})$ como também da margem lateral/circunferencial (1 mm) e da excisão total do mesorreto (ETM) difundiram-se as técnicas cirúrgicas com preservação esfincteriana.

$O$ interesse do cirurgião colorretal pela anastomose coloanal foi estimulado a partir do trabalho inicial de Parks (2) publicado em 1972. Lazorthes et al.(3) e Parc et al.(4) propuseram simultaneamente em 1986 o emprego do reservatório colônico após ressecções retais baixas no sentido de melhorar os resultados funcionais no pós-operatório imediato. Pelissier et al.(5),em 1992, realizaram a operação utilizando exclusivamente sutura mecânica, com o propósito de diminuir a contaminação intra-operatória. Em 1995, Soew-Choen e Goh (6) comprovaram em estudo prospectivo e randomizado, as vantagens funcionais do uso da bolsa colônica em $\mathrm{J}$, quando comparados à anastomose coloanal direta. Halböök et al.(7) em 1996, também em estudo prospectivo randomizado, demonstraram menores índices de complicações pós-operatórias e melhores resultados funcionais com a utilização do reservatório colônico em J.

O consenso geral é que a maioria dos cânceres do reto localizado na porção distal do terço inferior ( $<5 \mathrm{~cm}$ da borda anal) seja tratado por ressecção abdominoperineal do reto (RAP). Entretanto, com a aceitação recente de margem distal de ressecção menor do que $2 \mathrm{~cm}(8$ ) aumentou-se as indicações das operações de preservação esfincteriana. Por isso, alguns autores(9-11) propuseram recentemente a técnica de ressecção interesfinctérica, mesmo, em pacientes com câncer do reto invadindo o músculo esfíncter interno, já que o limite para a indicação parece ser mais circunferencial do que distal. Assim, a taxa de RAP vem progressivamente diminuindo.

A aceitação definitiva da cirurgia videolaparoscópica para o tratamento do câncer colorretal foi devido não só aos benefícios definitivos no curto prazo, principalmente, com relação à recuperação pós-operatória, mais também em função de uma melhor preservação da resposta imunológica e resultados a longo prazo de cura no mínimo equivalentes ao da cirurgia convencional.

A Sociedade Americana de Cirurgões Colorretais( ASCRS) e a Sociedade Americana de Cirurgiões Gastroentestinais(SAGES) vem estimulando o desenvolvimento de estudos para avaliar a segurança, a eficácia e os benefícios da cirurgia videolaparoscópica no câncer do reto, principalmente, depois da publicação, em 2007, dos resultados a longo prazo do importante estudo randomizado do grupo UK MRC CLASICC (12), que recomenda o emprego da cirurgia videolaparoscópica nas ressecções anteriores do reto e nas ressecções abdominoperineais. Eric Rullier (9) realizou pela primeira vez, em 2003, a técnica de ressecção interesfinctérica por videolaparoscopia. John Marks (13), da Universidade de Kentucky, em 2005 preconizou iniciar a operação pelo acesso interesfinctérico e completar pelo acesso videolaparoscópico. Ramos (14) em 1995 publicou nota técnica semelhante.

Em serviços especializados em cirurgia colorretal, a ressecção anterior ultrabaixa do reto com anastomose coloanal por duplo grampeamento é rotina para o tratamento de pacientes selecionados, portadores de câncer do terço inferior do reto. No entanto, tanto a reconstrução através do reservatório colônico quanto a utilização do acesso videolaparoscópico ainda não têm aceitação universal e dependem da seleção adequada dos pacientes e da experiência do cirurgião.

Este estudo tem como objetivo analisar os resultados do emprego da ressecção anterior ultra-baixa e interesfinctérica do reto com anastomose coloanal realizadas por videolaparoscopia para tratamento de pacientes com câncer da porção distal do reto inferior.

\section{CASUÍSTICA E MÉTODOS}

\section{Critérios para a escolha dos doentes}

De um total de 491 pacientes operados pelo acesso videolaparoscópico, no período de janeiro 1992 a maio de 2009, foram selecionados para esse estudo prospectivo 13 doentes com câncer da porção distal do reto inferior. Os 240 pacientes com doença benigna, os 77 com câncer do cólon, os 52 com câncer do reto superior, os 58 com câncer do reto médio, e os 51 com câncer do reto inferior que foram submetidos à 
Tratamento Cirúrgico do Câncer da Porção Distal do Têrço Inferior do Reto pela Ressecção Anterior Ultrabaixa e Interesfinctérica com Anastomose Coloanal por Videolaparoscopia José Reinan Ramos e Cols. ressecção abdominoperineal do reto foram excluídos do estudo. Nenhum paciente com tumor T4 foi selecionado. Os pacientes foram operados pelo mesmo cirurgião (JRR) nos Serviços de Coloproctologia do Hospital do Andaraí e do Hospital da Lagoa e nos Hospitais Samaritano, São Lucas e Casa de Saúde São José (Rio de Janeiro).

Na tabela 1 está demonstrado as principais características dos pacientes (idade e sexo), do tumor (localização) e do tratamento pré-operatório (quimiorradioterapia).

A idade oscilou entre 39 e 80 anos com uma média de 63 anos.

Quanto ao sexo, 9 doentes $(69,3 \%)$ pertenciam ao sexo feminino e, 4 doentes (30,7\%), ao masculino.

Todos os tumores estavam localizados na porção distal do reto inferior, até $4 \mathrm{~cm}$ acima da linha pectínea. A biópsia confirmou adenocarcinoma em todos os tumores.

A quimiorradioterapia neo-adjuvante (5FU-Leu $+45 \mathrm{G})$ foi utilizada em 8 doentes $(61,4 \%)$.Todos tiveram resposta parcial.

\section{AVALIAÇÃO PRÉ-OPERATÓRIA}

A avaliação pré-operatória pélvica foi realizada com toque retal, retossigmoidoscopia e ressonância magnética em 10 pacientes e tomografia computadorizada nos outros 3 .
A colonoscopia realizado em todos os pacientes foi o exame utilizado para excluir lesões síncrônicas do cólon. A tomografia computadorizada hepática e a ressonância magnética hepática foram usadas para excluir metástase no fígado e $\mathrm{o}$ raio $\mathrm{X}$ de tórax para excluir metástase pulmonar.

\section{TÉCNICA OPERATÓRIA}

Todos os doentes foram submetidos a tratamento operatório eletivo.

A técnica operatória empregada esta publicada na íntegra na nota técnica de Ramos JR (15).Os principais passos da operação são:

1 - Posição do Paciente (Figura 1).

2 - Posicionamento do Equipamento e Equipe(Figura 2)

3 - Posicionamento dos Trocartes e Exploração da Cavidade Abdominal (Figura 3)

4 - Exposição do Campo Operatório

5 - Ligadura dos Vasos Mesentéricos Inferiores pelo acesso medial (Figura 4) Sigmóide

6 - Mobilização do Ângulo Esplênico e do Colon

7 - Excisão total do mesorreto, preservação dos nervos pélvicos e mobilização do reto pela técnica de Rullier (Figuras 5,6,7,8,9,10).

(Figura 11)

Tabela 1 - Dados dos pacientes, do tumor e do tratamento pré-operatório.

\begin{tabular}{lcccc}
\hline Identificação & Idade & Sexo & Distância Tumor - Linha Pectínea & Quimioradioterapia Pré-Op \\
\hline MAL & 80 & $\mathrm{~F}$ & $4,0 \mathrm{~cm}$ & Não \\
AMAO & 56 & $\mathrm{~F}$ & $1,5 \mathrm{~cm}$ & Sim \\
NS & 58 & M & $1,5 \mathrm{~cm}$ & Sim \\
VE & 65 & M & $0,5 \mathrm{~cm}$ & Sim \\
NS & 71 & M & $3,0 \mathrm{~cm}$ & Sim \\
MTMTS & 39 & $\mathrm{~F}$ & $0,5 \mathrm{~cm}$ & Nâo \\
MC & 55 & $\mathrm{~F}$ & $3,0 \mathrm{~cm}$ & Sim \\
JS & 58 & M & $1,0 \mathrm{~cm}$ & Sim \\
TGS & 61 & $\mathrm{~F}$ & $3,5 \mathrm{~cm}$ & Não \\
MJPS & 49 & $\mathrm{~F}$ & $3,0 \mathrm{~cm}$ & Sim \\
MS & 67 & $\mathrm{~F}$ & $2,0 \mathrm{~cm}$ & Não \\
MP & 63 & $\mathrm{~F}$ & $1,5 \mathrm{~cm}$ & Não \\
JPS & 60 & $\mathrm{~F}$ & $1,0 \mathrm{~cm}$ & Sim \\
\hline
\end{tabular}


Rev bras Coloproct Julho/Setembro, 2009
Tratamento Cirúrgico do Câncer da Porção Distal do Têrço Inferior do Reto pela Ressecção Anterior Ultrabaixa e Interesfinctérica com Anastomose Coloanal por Videolaparoscopia José Reinan Ramos e Cols.
Vol. 29
9 - Ressecção interesfinctérica (RI) e anastomose coloanal (Figuras 12,13,14,15,16)

\section{RESULTADOS}

Não ocorreu complicação intra-operatória nem conversão nesse grupo de pacientes. Três pacientes $(23,1 \%)$ tiveram complicações no pósoperatório.Uma importante devido a fístula anastomótica $(7,7 \%)$ e abscesso pélvico que foi drenado para o ânus e o paciente permanece com estoma de proteção e duas outras complicações menores (seroma e íleo pós-operatório).

A mortalidade, com o emprego dessa técnica nesse grupo de pacientes foi nula. A alta hospitalar ocorreu até o 7.o dia de pós-operatório para 8 pacientes $(61,5 \%), 4$ dos quais no quinto dia; os outros 5 tiveram alta hospitalar no 9.0,10.10.12 e 15 dias de pósoperatório.

O exame anatomopatológico comprovou adenocarcinoma em todas as peças operatórias. Dois tumores foram classificados como T1NoMo, dois como T2N0M0, três como T3NoMo, três como T2N1Mo e três como T3N1Mo. O número de linfonodos examinados variou de 7 a 19 (média de 13 linfonodos por peça). A margem distal variou de $0,5 \mathrm{~cm}$ a $3,5 \mathrm{~cm}$ (média de $1,5 \mathrm{~cm}$ ). A margem circunferencial foi positiva em um caso $(7,7 \% ; 0,5 \mathrm{~mm})$. (Figuras 17,18,19).

A avaliação clínica dos sintomas evacuatórios mostrou uma melhora evolutiva até 18 meses após o fechamento da íleostomia. Evacuação fracionada foi relatada por 11 pacientes (91\%). Incontinência fecal

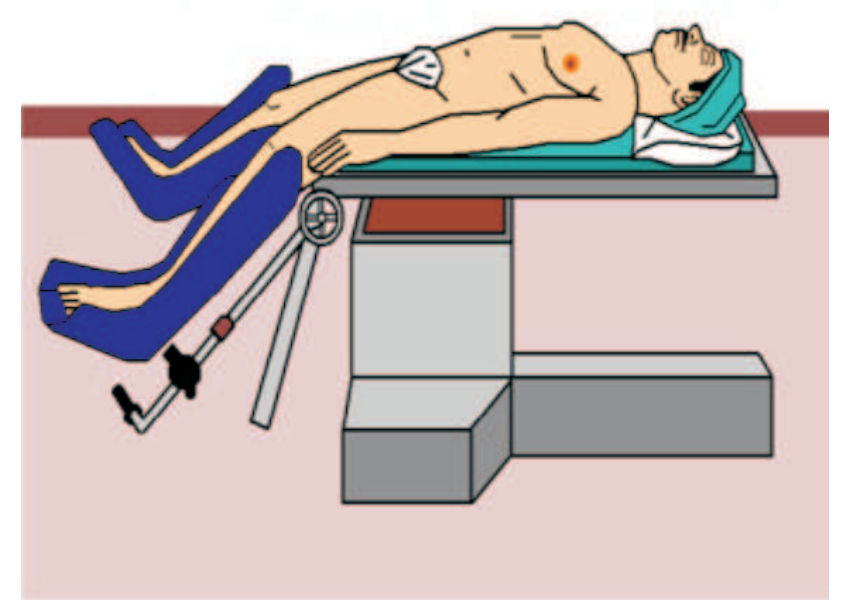

Figura 1 - Posição do paciente. foi relatado por 5 pacientes (41\%) sendo que um deles utiliza proteção anal (frauda) após 6 meses de operado. Quatro foram irradiados no pré-operatório (4/ $7=57 \%$ ). No grupo não irradiado (5 pacientes) ocorreu um caso de incontinência(20\%). Onze pacientes $(84 \%)$ estão satisfeitos com a operação. Um paciente continua ileostomizado $(7,7 \%)$.

Em um seguimento pós-operatório médio de 30 meses ocorreu uma recidiva local $(7,7 \%)$ diagnosticada em uma paciente no 16.0 mês e metástase pulmonar em duas pacientes $(15,4 \%)$ diagnosticadas no 18.0 e 22.o mês. As três pacientes tinham tumores estádio III e faleceram da doença. Dez pacientes $(77 \%)$ estão sem doença em acompanhamento ambulatorial.

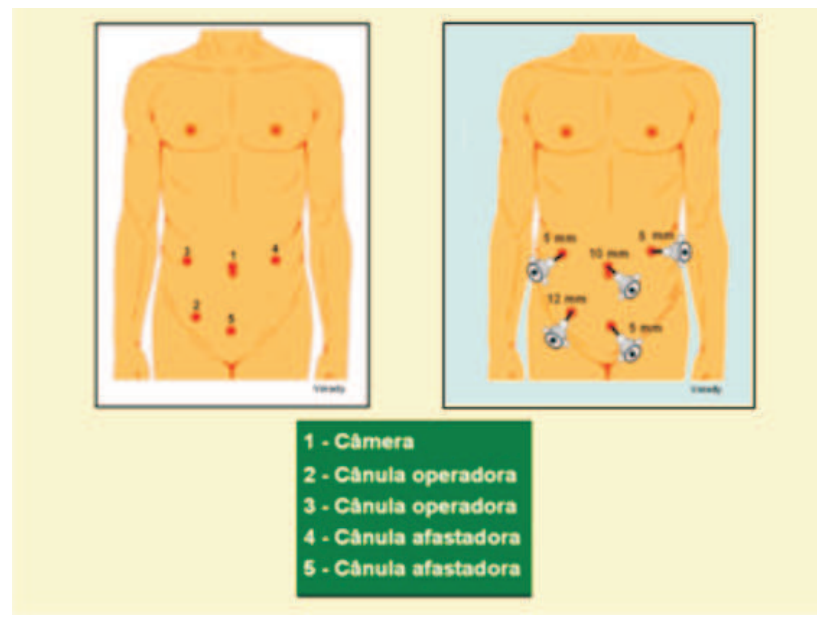

Figura 2 - A - Numeração das cânulas; B - Posicionamento dos trocartes; $C$ - Função da cânulas.

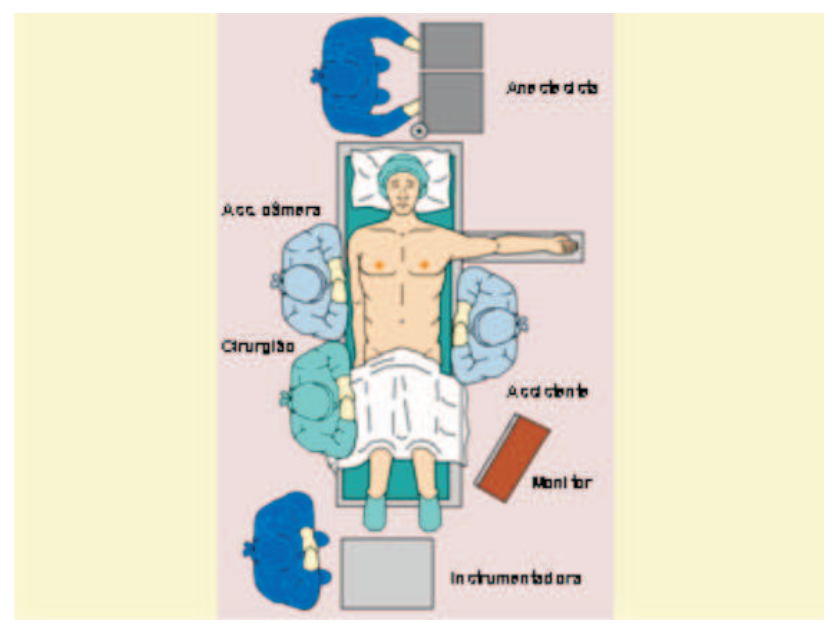

Figura 3 - Posição da equipe. 

José Reinan Ramos e Cols.

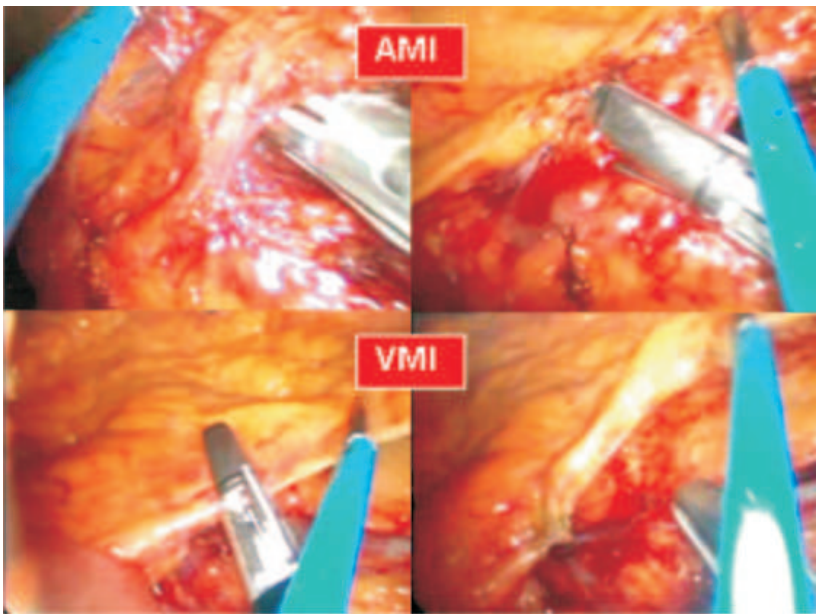

Figura 4 - Identificação e selagem alta dos vasos mesentéricos inferiores. A e $B$ - Artéria mesentérica inferior. $C$ e $D$ - Veia mesentérica inferior.

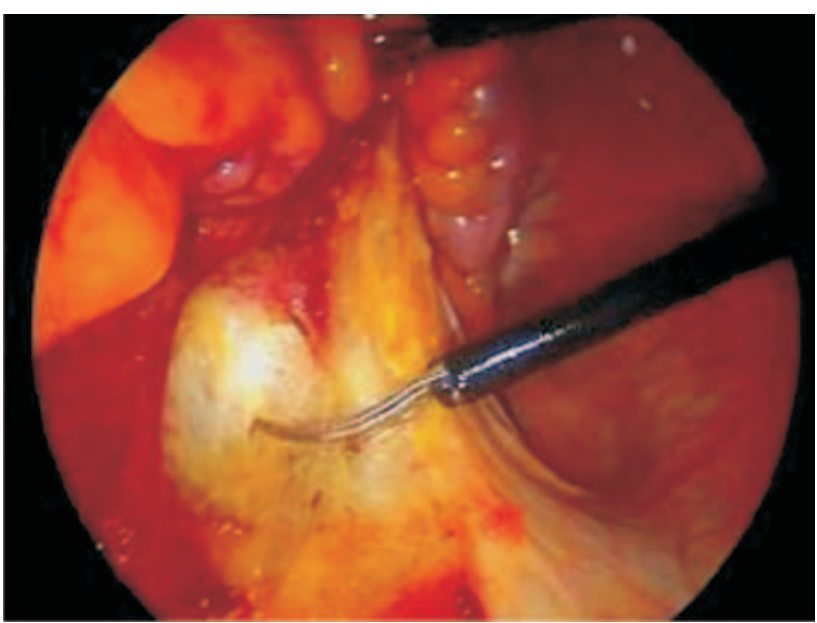

Figura 5 - Excisão total do mesorreto (Técnica de Rullier). Tempo 1 da Dissecção Retal Alta-Dissecção posterior alta (Referência anatômica: espaço pré-sacral/promontório).

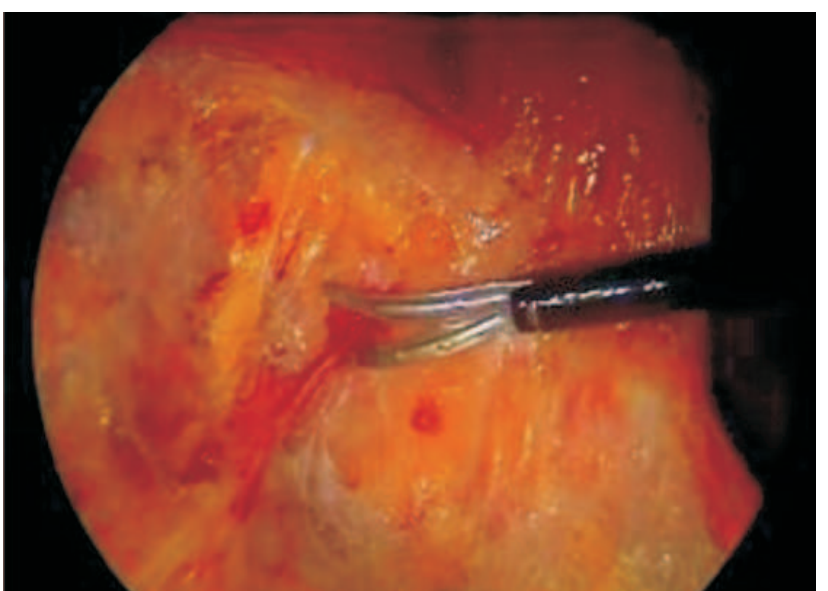

Figura 6 - Excisão total do mesorreto (Técnica de Rullier). Tempo 2 da Dissecção Retal Alta-Dissecção lateral alta (Referência anatômica: nervos hipogástricos).

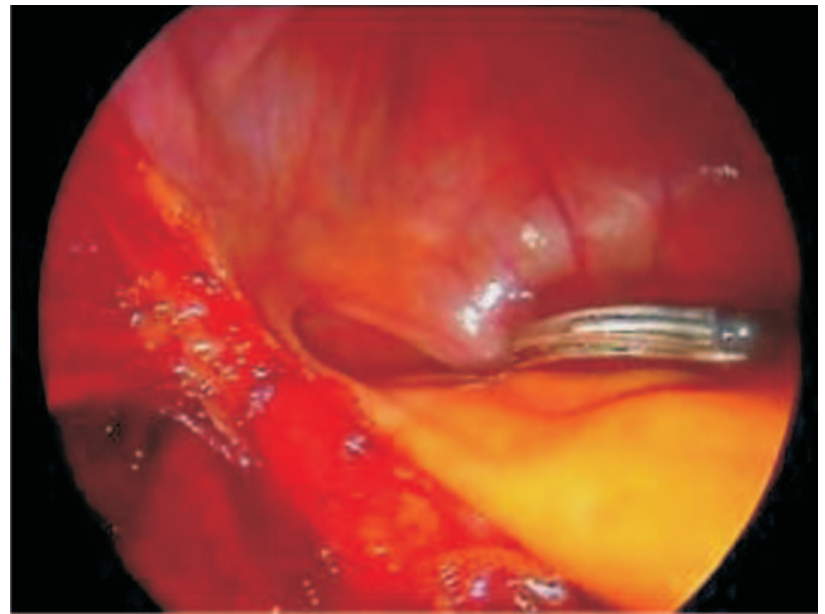

Figura 7 - Excisão total do mesorreto (Técnica de Rullier). Tempo 3 da Dissecção Retal Alta-Dissecção anterior alta(referência anatômica: vesículas seminais ou a cúpula vaginal.

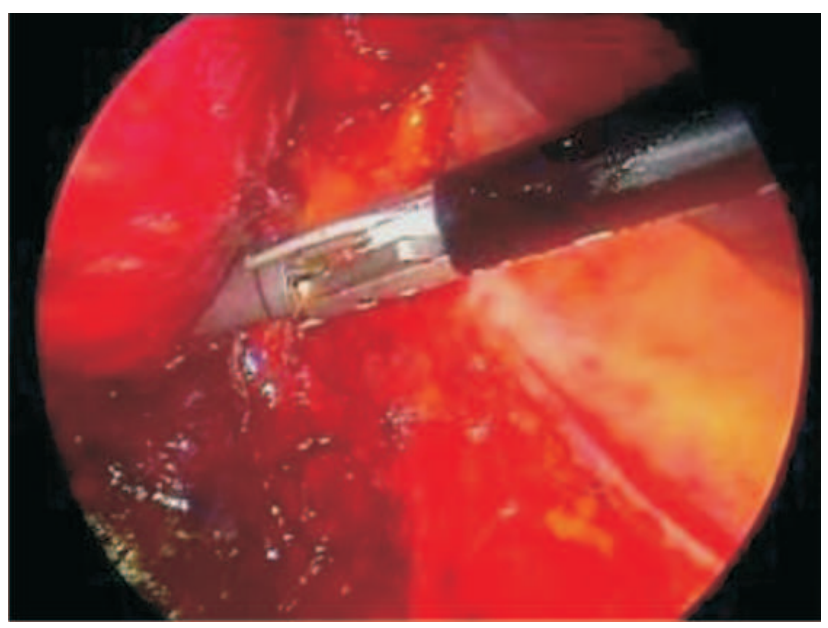

Figura 8 - Excisão total do mesorreto (Técnica de Rullier). Tempo 4 da Dissecção Retal Baixa-Dissecção Lateral baixa (Referência anatômica: nervos o plexo pélvico parassimpático).

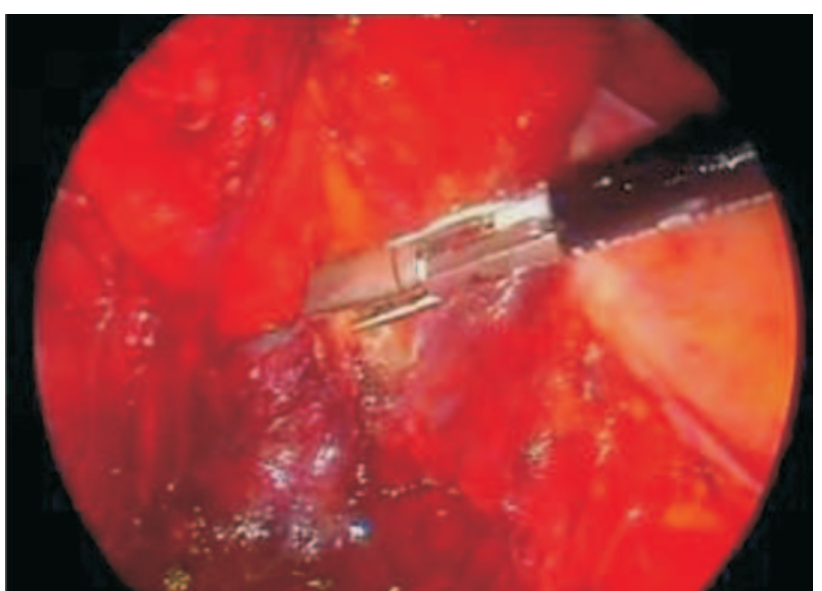

Figura 9 - Excisão total do mesorreto (Técnica de Rullier). Tempo 5 da Dissecção Retal Baixa-Dissecção posterior baixa (referência anatômica: músculos elevadores do ânus). 
Rev bras Coloproct Julho/Setembro, 2009
Tratamento Cirúrgico do Câncer da Porção Distal do Têrço Inferior do Reto pela Ressecção Anterior Ultrabaixa e Interesfinctérica com Anastomose Coloanal por Videolaparoscopia José Reinan Ramos e Cols.

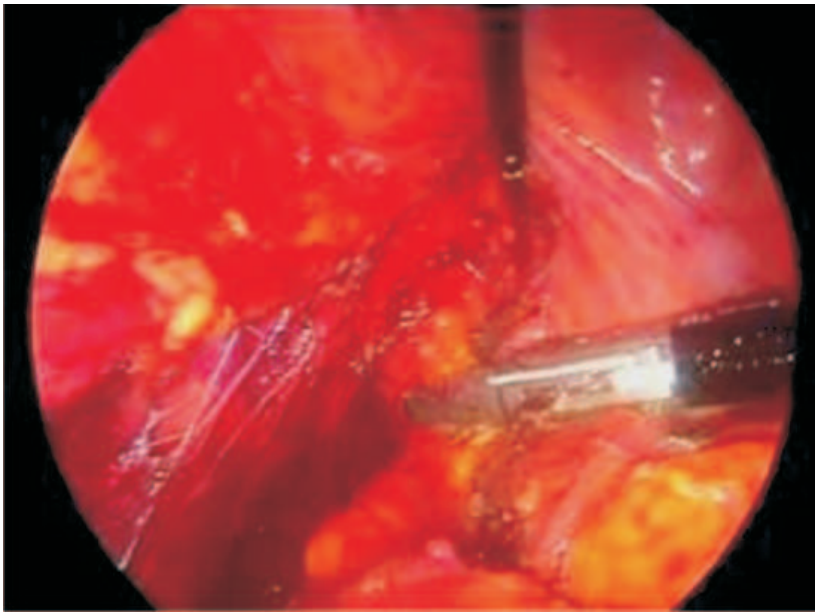

Figura 10 - Excisão total do mesorreto (Técnica de Rullier). Tempo 6 da Dissecção Retal Baixa-Dissecção anterior baixa (referência anatômica: a fascia de Denonvilliers e a próstata ou vagina).

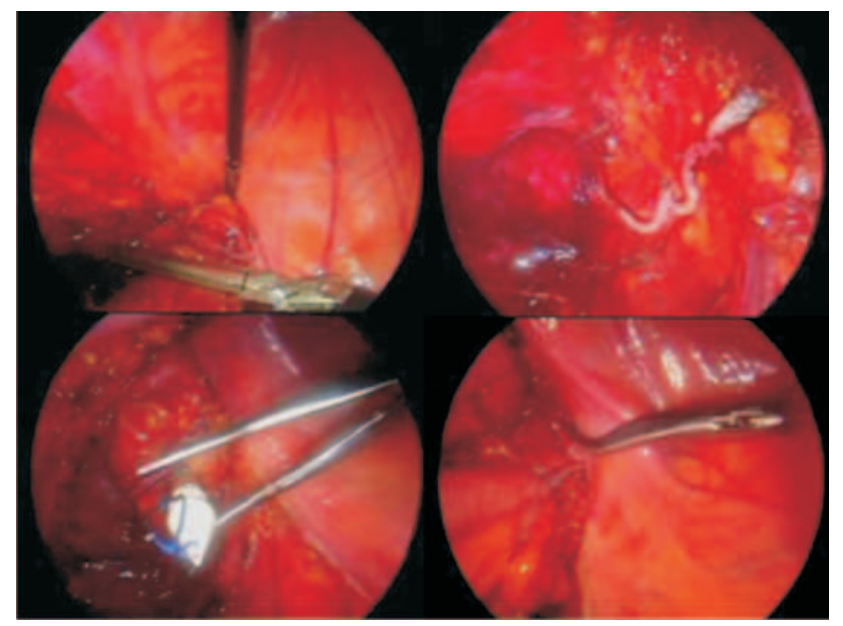

Figura 11 - Anastomose coloanal via abdominal. A - Endogrampeamento retal baixo com grampeador articulado. B - Linha de grampeamento no topo do canal anal. $C$ - Perfuração do côto retal para conexão da ogiva. D - Final da anastomose coloanal latero-terminal.

\section{DISCUSSÃO}

O reservatório colônico em $\mathrm{J}$ classicamente é indicado para a reconstrução do trânsito intestinal após a ressecção de tumores localizados no reto extraperitonial, especialmente, na porção distal do têrço médio do reto, e no têrço inferior, quando não há invasão da musculatura esfincteriana ou dos musculos elevadores do ânus. O cólon sigmoide proximal pode ser usado para a realização do reservatório em $\mathrm{J}$ sem prejuízo da radicalidade oncológica. $\mathrm{O}$ tamanho da bolsa colônica $(5 \mathrm{~cm})$ é mais importante do que o segmento
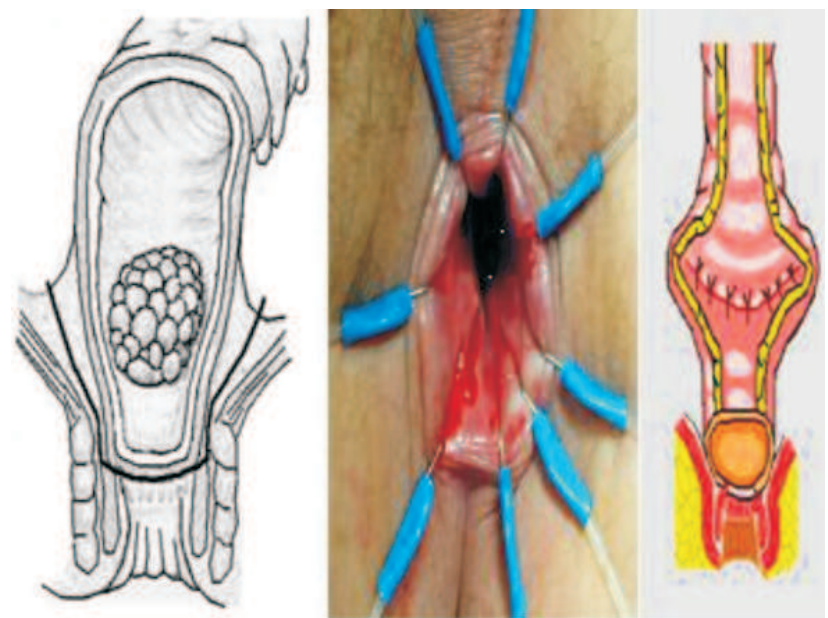

Figura 12 - A-Demonstração da secção do reto distal (Traço preto) pelo acesso interesfinctérico com ressecção parcial do esfincter interno. B - Exposição transanal da margem distal.c-Demostração da coloplastia transversa.

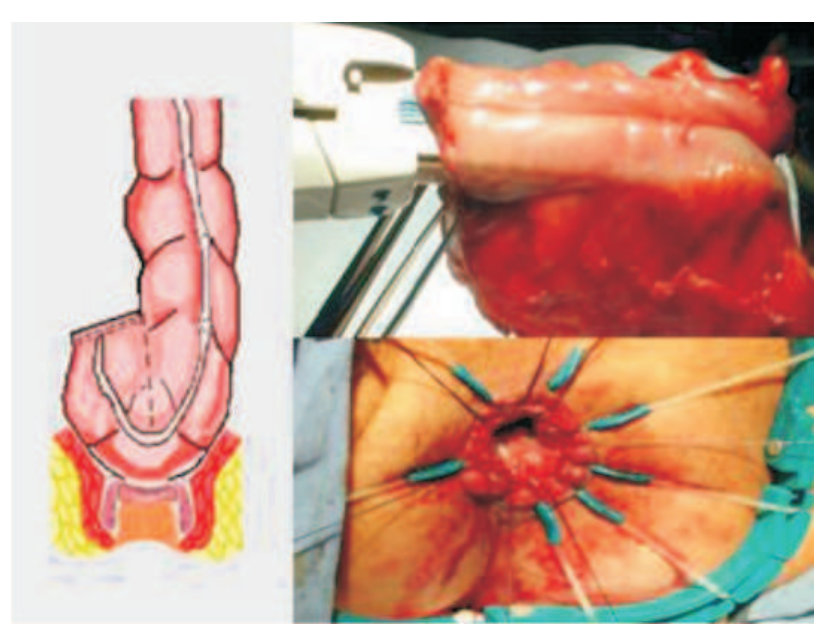

Figura 13 - A - Demostração da bolsa colônica em J de $5 \mathrm{~cm}$. BAnastomose latero-lateral grampeada para confecção da bolsa em J.c-Anastomose coloanal manual.

colônico utilizado. Essa medida confere não só um adequado reservatório como também propicia um bom esvaziamento(16-17).

Os resultados funcionais da $\mathrm{BCJ}$ de $5 \mathrm{~cm}$ são melhores do que os da anastomose coloanal direta, o que se deve, provavelmente, mais à diminuição da motilidade do cólon do que à capacidade volumétrica da bolsa. No entanto, alguns fatores de ordem técnica podem inviabilizar a realização da BCJ, como: impossibilidade de mobilização do cólon; comprimento do cólon inadequado; doença diverticular; pelve muito estreita; e cólon adiposo, entre outros. Z'Graggen et 

José Reinan Ramos e Cols.

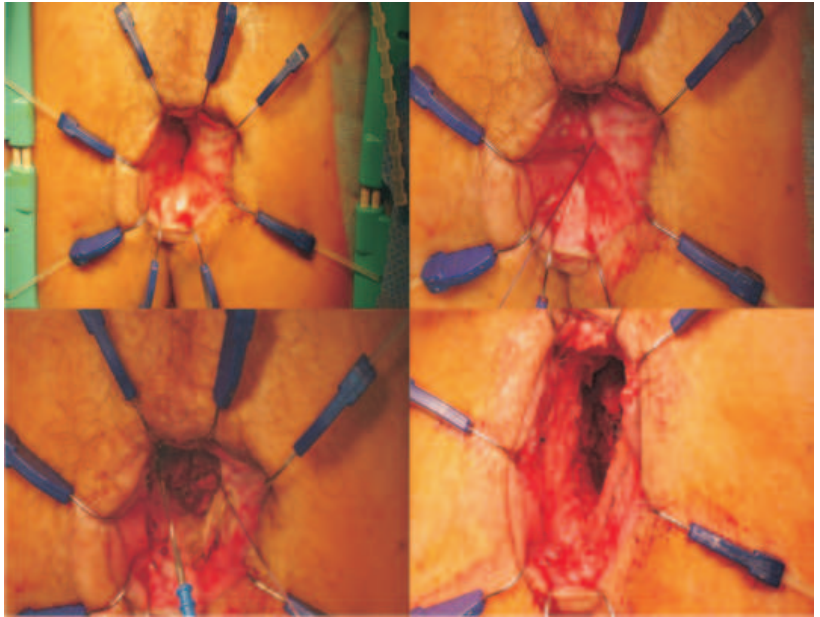

Figura 14 - A - Exposição docanal anal com o afastador de Lonestar. B - Fechamento com sutura em chuleio abaixo da linha pectínea. $C$ - Inicio da ressecção interesfinctérica. D - Final da ressecção interesfinctérica com excisão do esfíncter interno.

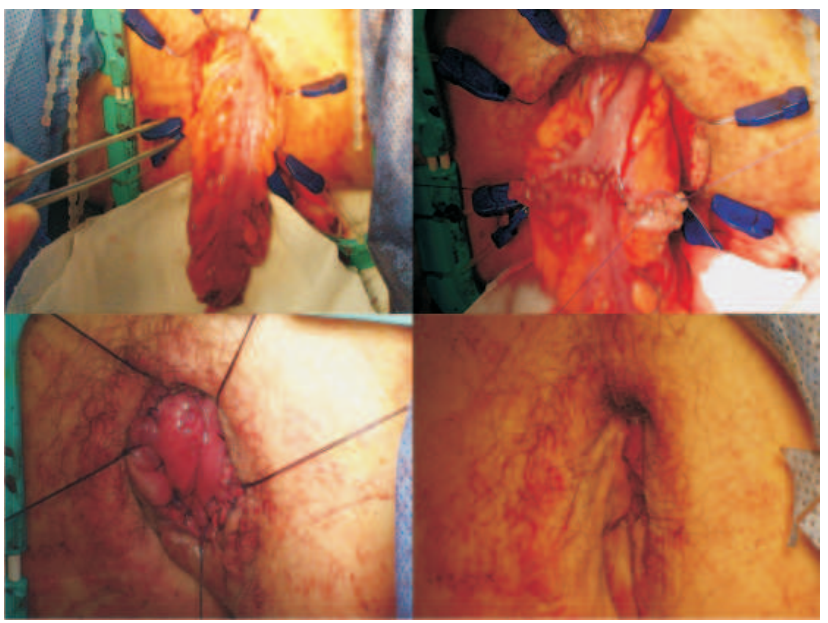

Figura 15 - Coloplastia. A - Exposição do cólon abaixado. B Coloplastia transversa em chuleio. $C$ - Anastomose coloanal em plano único. $D$ - Aspecto final do ânus.

al.(18) e Fazio et al (19) descreveram a técnica de coloplastia transversa, similar à piloroplastia tipo Heinecke-Mikulicz, e a propuseram como método alternativo à BCJ. .Ho et al.(20), entretanto, relataram maior incidência de fístula anastomótica ( 0 versus $15,9 \% ; \mathrm{p}=0.0121)$ e de complicações $(9.1 \%$ versus $31.8 \% ; \mathrm{p}=0.02287)$ nos pacientes com coloplastia. Huber et al.(21) relataram resultados clínicos (complicações pós-operatórias) e funcionais safisfatórios, após 6 meses, quando utilizaram a anastomose colo-anal direta latero-terminal sem bolsa. Os três reservatórios apresentam resultados funcionais semelhantes(22) Acreditamos que os menores índices de fístulas obser-

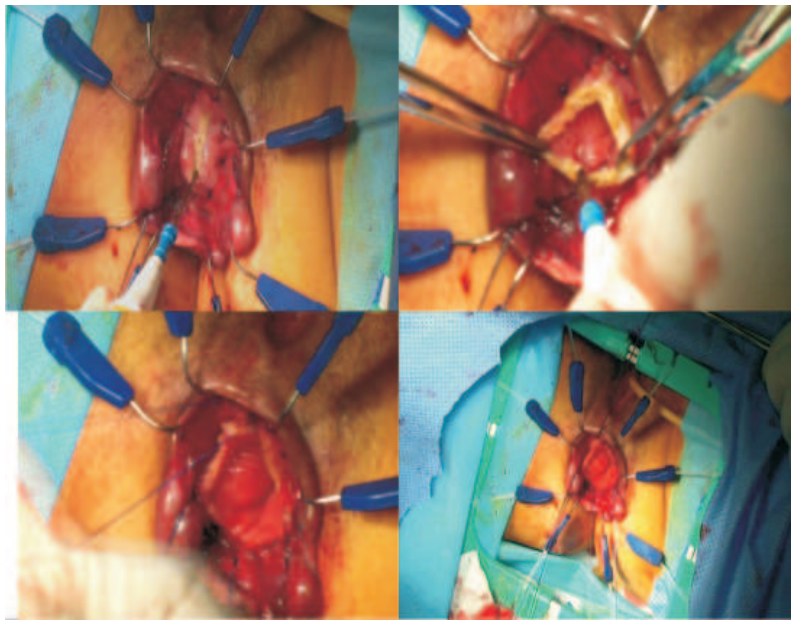

Figura 16 - Anastomose Latero-lateral. A - Exposição e abertura do cólon. $B$ - Final da abertura na borda antemesentérica. $C$ Anastomose coloanal. D - Anastomose coloanal com pontos separados.

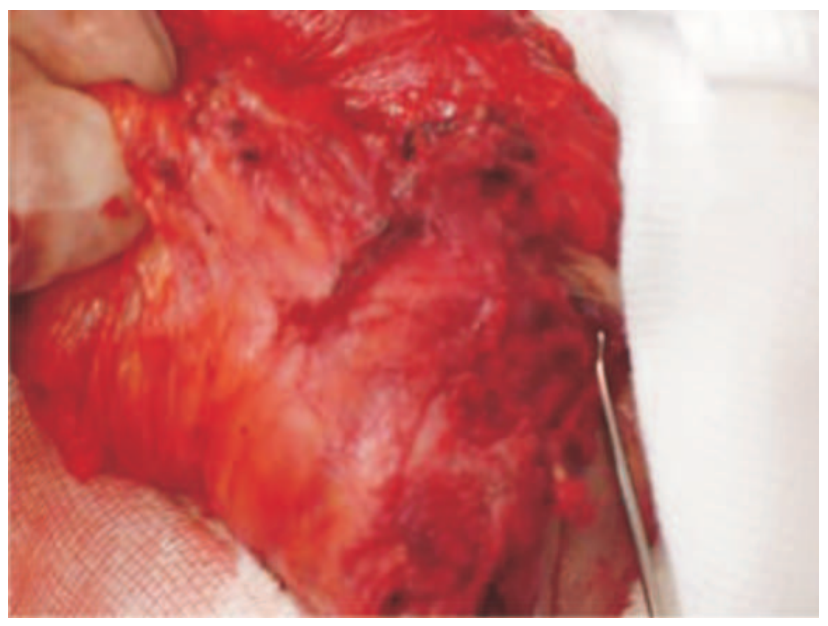

Figura 17 - Excisão total do mesorreto com margem circunferencial negativa.

vados nas anastomoses com BCJ estejam relacionados à melhor vascularização do ápice da bolsa colônica, quando comparada à vascularização da porção distal do cólon na anastomose direta como mostra estudo ( 23) de avaliação do fluxo sanguíneo através de laser Doppler.

Os efeitos tardios da irradiação pré-operatória nos resultados funcionais dos pacientes com câncer do reto distal, tratados com ressecção anterior ultra-baixa e bolsa colônica em J, ainda não são totalmente conhecidos. Estudos sugerem que a anastomose da bolsa colônica em J deva ser realizada no canal anal e não no reto residual irradiado. Também é aconselhável evi- 


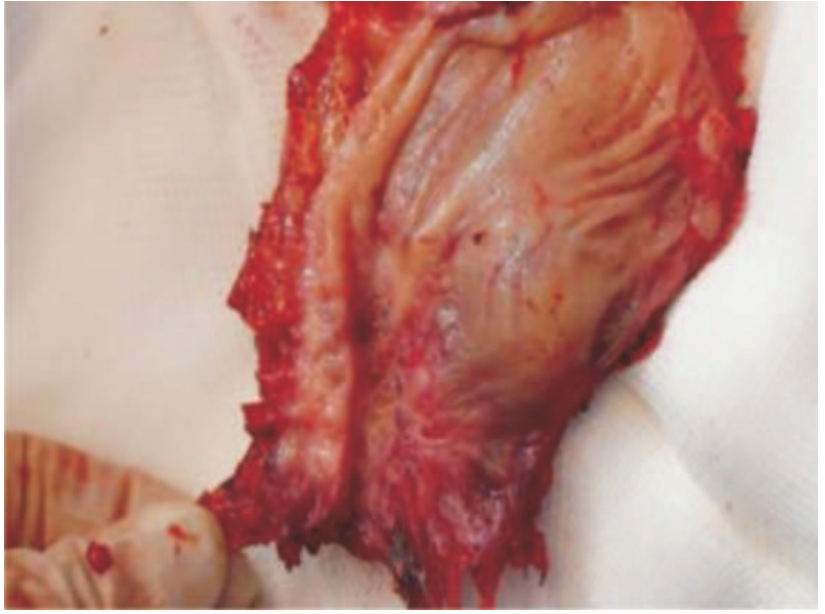

Figura 18 - Peça operatória aberta com margem distal livre de 0,5 cm. Peça irradiada.

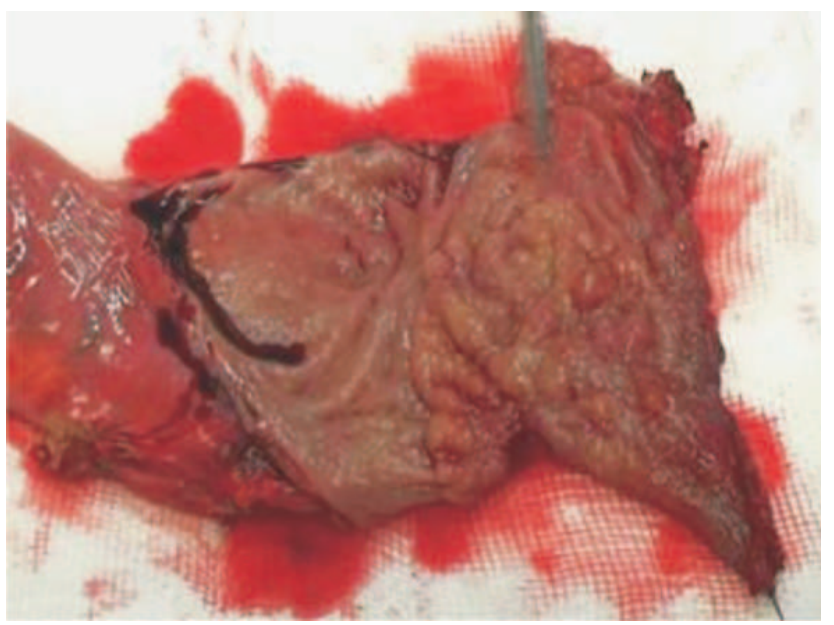

Figura 19 - Peça operatória aberta com margem distal livre de 0,5 cm. Peça não irradiada.

tar a irradiação dos esfíncteres anais, já que os danos da radioterapia são cumulativos e a disfunção dos esfíncteres pode ser progressiva no decorrer dos anos. No estudo da Cleveland Clinic da Florida (24), quando comparados os grupos de cirurgia exclusiva versus cirurgia associada à radioquimioterapia pré ou pós-operatória, observou-se diferença nos seguintes parâmetros avaliados : diminuição da pressão de repouso do canal anal ( $24 \%$ versus $51 \%$; $\mathrm{p}=0.03)$, alteração da continência anal $(\mathrm{p}<0.001)$, utilização de "pads" $(18 \%$ versus $53 \% ; \mathrm{p}=0.02)$ e problemas evacuatórios $(\mathrm{p}<0.001)$.

No nosso estudo, também foi observado resultados funcionais clínicos piores nos 7 pacientes submetidos à quimiorradioterapia pré-operatória quando comparados aos 5 pacientes que foram tratados somente com cirurgia (incontinência: $1 / 5-20 \%$ versus 4/ $7-57 \%)$.

Rullier et al.(25) avaliaram os resultados da quimiorradioterapia pré-operatória em 332 pacientes tratados com câncer do reto. Comparado com cirurgia exclusiva a quimiorradioterapia diminuiu não só o número de linfonodos recuperados nas peças (17 versus 13; $\mathrm{P}<0.001)$ como também o número de linfonodos positivos (2.3 versus $1.2 ; \mathrm{P}=0.001)$. Embora, a quimiorradioterapia pré-operatória diminuisse em $24 \%$ o numero de linfonodos recuperados e em $48 \%$ o número de linfonodos positivos, a sobrevida não foi influenciada pelo número de linfonodos recuperados nas peças irradiadas. Habr-Gama et al.(26) sugerem tratamento não operatório com seguimento rigoroso em casos de resposta completa à quimiorradioterapia neoadjuvante.

Rullier et al. (8) empregaram cirurgia conservadora (ressecção interesfinctérica e anastomose coloanal) em 92 pacientes com câncer do reto distal até $4,5 \mathrm{~cm}$ da margem anal quando não havia infiltração do músculo esfíncter externo. A mortalidade foi nula e as complicações ocorreram em $27 \%$ dos casos. A ressecção microscópica foi completa (R0) em $89 \%$, com $98 \%$ de margem distral negativa e $89 \%$ de margem circunferencial negativa. A recidiva local em 24 meses foi de $2 \%$.

Saito et al (10) investigaram os resultados oncológicos e funcionais em 228 pacientes com câncer do reto localizados até $5 \mathrm{~cm}$ acima da borda anal que foram submetidos à ressecção interesfinctérica pela técnica aberta em sete instituições do Japão. A taxa de mortalidade foi de $0,4 \%$ e a de complicações pós-operatória de $24 \%$. A recidiva local em 3 anos foi de 5,8\% e a sobrevida livre de doença em 5 anos foi $83 \%$. A continência anal após 2 anos foi boa em $68 \%$ dos pacientes e ruim em 7\%, principalmente, nos pacientes em que as ressecções dos esfíncteres foram extensas.

$\mathrm{Na}$ avaliação de 278 pacientes submetidos à anastomose coloanal, 173 com ressecção interesfinctérica Portier et al. (27) relataram 10,6\% de recorrência local em 5 anos para o grupo da RI e 6.7 $\%$ para as anastomoses coloanais sem RI $(\mathrm{P}=0.405)$, e sobrevida global em 5 anos similares (86\% versus 80\%). Weiser et al. (11) do Departamento de Cirurgia do Memorial Sloan-Kettering Cancer Center avaliaram os resultados de três tipos de cirurgias: Ressecção anterior ultrabaixa com anastomose coloanal grampe- 
Tratamento Cirúrgico do Câncer da Porção Distal do Têrço Inferior do Reto pela Ressecção Anterior Ultrabaixa e Interesfinctérica com Anastomose Coloanal por Videolaparoscopia José Reinan Ramos e Cols. ada (41 casos), com ressecção interesfinctérica e anastomose manual (44 casos) e ressecção abdominoperineal do reto (63 casos). Os tumores estavam localizados no reto distal a menos de $6 \mathrm{~cm}$ da borda anal. Todos os pacientes receberam quimiorradioterapia pré-operatória. A ressecção foi completa com margens histológicas negativas em $92 \%$ das peças; a margem circunferencial foi positiva em $5 \%$ das ressecções interesfinctéricas e em $13 \%$ das RAP. A margem distal foi positiva em 5\% dos casos de ressecção interesfinctérica. A recidiva local em 47 meses foi de $5 \%$, sendo a maioria (6 casos) no grupo da RAP. A recorrência estimada e a sobrevida foram de $85 \%, 83 \%$, e $47 \%$, no grupo das anastomoses grampeadas, da ressecção interesfinctérica e da RAP, respectivamente $(\mathrm{P}=0.001)$. $\mathrm{O}$ índice de recidiva local e à distância em 3 anos encontrado por Akasu et al.(28) nas ressecções interesfinctéricas foi de $6 \%$ e $13 \%$, respectivamente. $\mathrm{O}$ risco de recidiva local está relacionado à margem de ressecção e o de metástase à distância ao estatus linfonodal.

Ito el al.(29) concluíram em estudo com 96 pacientes que a quimiorradioterapia é o fator de risco de maior impacto negativo nos resultados funcionais dos pacientes submetidos à ressecções interesfinctéricas, apesar da ressecção do esfíncter interno.Tilney e Tekkis (30) reviram 21 trabalhos de 13 serviços e selecionaram 612 pacientes. A mortalidade operatória após ressecção interesfinctérica foi de $1,6 \%$ e de fístula anastomótica de $10.5 \%$. A taxa de recidiva local foi de $9.5 \%$ com uma sobrevida média em 5 anos de $81.5 \%$. Resultados semelhantes aos encontrados na nossa casuística (Taxa de fístula anastomótica $=7,7 \%$; de recidiva local=7,7\%; de metástase à distância 15,4\%; e, de sobrevida $=77 \%$ ).

Tsang et al.(31) avaliaram os resultados pósoperatórios de 105 pacientes submetidos à excisão total do mesorreto e anastomose coloanal por videolaparoscopia.. A anastomose ficou em média a $3,9 \mathrm{~cm}$ da margem anal. Conversão ocorreu em 2 casos e 6 pacientes foram reoperados por complicações. A mortalidade foi nula. A recidiva local foi de $8.9 \%$.

Marks et al.(13) compararam dois grupos de pacientes com câncer do reto distal tratados por ressecção interesfinctérica pela via laparoscópica (30 casos) e pela via aberta (35 casos). A recorrência local, num seguimento de 18 meses ,foi diagnosticada em 1 paciente, do grupo laparoscópico (3,3\%) e metástase à distância em 8 pacientes, 7 (20\%) do grupo de cirurgia aberta. Evitou-se a colostomia definitiva em $89 \%$ dos pacientes.

Em doze (92,3\%) dos pacientes estudados no nosso trabalho também não foi necessário o uso do estoma definitivo.

Laurent et al. (32) acompanharam 200 pacientes com câncer do reto médio e inferior que foram tratados por excisão total do mesorreto por videolaparoscopia (79 anastomoses colorretais e 121 anastomoses coloanais). A taxa de conversão foi de $15.5 \%$ (31 operações) e as complicações pós-operatórias ocorreram em 50 pacientes $(25 \%)$.

Estes resultados são semelhantes aos encontrados na nossa série, cuja taxa de complicações pósoperatória foi de $25 \%$, e nula para conversão e mortalidade.

Os resultados oncológicos, inferidos a partir das taxas de cura e de recidiva, não dependem do tipo de recontrução, mas sim da experiência do cirurgião, da qualidade técnica da ressecção, do estádio da doença, do uso racional de quimioterapia e radioterapia e das condições de seguimento. Uma melhor definição da resposta tumoral às terapias neoadjuvantes, através da biologia molecular, deverá facilitar o desenvolvimento de estratégias individuais para o tratamento do câncer do reto.

\section{CONCLUSÕES}

A análise dos resultados obtidos com o emprego da ressecção anterior ultrabaixa e interesfinctérica do reto e anastomose coloanal por videolaparoscopia no tratamento de 13 pacientes com câncer localizado na porção distal do terço inferior do reto, apesar da pequena casuística, permitiu as seguintes conclusões :

1. A técnica empregada mostrou ser viável e segura, pois apresentou baixo índice de complicação pós-operatória e mortalidade nula.

2. O emprego dessa técnica permitiu período de internação hospitalar pós-operatório curto.

3. Apesar dos resultados funcionais com avaliação clínica regular, evitou-se a colostomia definitiva em $92,3 \%$ dos doentes.

4 A utilização dessa técnica não comprometeu os resultados oncológicos num período médio de 30 meses de seguimento. 
Rev bras Coloproct Julho/Setembro, 2009
Tratamento Cirúrgico do Câncer da Porção Distal do Têrço Inferior do Reto pela Ressecção Anterior Ultrabaixa e Interesfinctérica com Anastomose Coloanal por Videolaparoscopia José Reinan Ramos e Cols.

\begin{abstract}
Purposes: The main purpose of this study was to evaluate the postoperative complications, the length of the hospital stay, the clinic functional results, and the oncological outcome at 2,5 years follow-up of patients with very low rectal cancer treated by laparoscopic coloanal anastomosis and intersphincteric resection. Patients and Methods: 491 patients were treated by laparoscopic colorectal surgery, 13 of 172 with rectal cancer were selected and prospectively evaluated. All with very low rectal cancer, 9 female. No patient $\mathbf{T} 4$ or with complete response to quimioirradiation was selected. Quimioirradiation was used in 8 patients. RESULTS: The postoperative complication rate was $23,1 \%$ and the anastomotic fistulas rate was $7,7 \%$. No patients died postoperatively. $61,5 \%$ of the patients were discharged before 7 days of hospital stay. The median number of harvested lymphnodes was 13 . Mean distal tumor-free margin was $1,5 \mathrm{~cm}$. Circumferential margin was positive in $1 \mathrm{case}(7,7 \%)$. Fecal incontinence was related in $41 \%$ of the patients and fracionned evacuations in $91 \%$. Eleven patients $(84 \%)$ have related good quality of life. One patient is with definitive stoma $(7,7 \%)$. With median follow-up of 30 months, there were one local recurrence $(7,7 \%)$ and two cases of lung metastases $(15,4 \%)$. All, the three patients died of the diseases. Ten patients are survived (77\%) without disease. CONCLUSIONS: Analysis of results led to the following conclusions: a) The technique employed is safe and have presented low rate of complication and no mortality; b) The use of this technique have permited short length of the hospital stay; c) Functional results were regular, but colostomy was avoid in 92,3\% of the patients; d) The use of this technique does not compromise the oncological outcome at a median follow-up of 30 months.
\end{abstract}

Key words: Intersphincteric Resection, Rectal Cancer, Laparoscopy.

\section{REFERÊNCIAS}

1. Miles EW. A method of performing abdominoperineal excision for carcinoma of the rectum and of the terminal portion of the pelvic column. Lancet. 1908; 2: 1812-1813.

2. Parks AG. Transanal technique in low rectal anastomosis. Proc R Soc Med 1972;65:975-6.

3. Lazorthes F, Fages P, Chiotasso P, Lemozy J, Bloom E. Resection of the rectum with construction of a colonic reservoir and colo-anal anastomosis for carcinoma of the rectum. Br J Surg 1986;73:136-8.

4. Parc R, Tiret E, Frileux P, Moszkowski E, Loygue J. Resection and colo-anal anastomosis with colonic reservoir for rectal carcinoma. Br J Surg 1986;73:139-41.

5. Pélissier EP, Blum D, Bachour A, Bosset JF. Functional resultas of coloanal anastomosis with reservoir. Dis Colon Rectum 1992;35:843-6.

6. Seow-Choen F, Goh HS. Prospective randomized trial comparing $\mathrm{J}$ colonic pouch-anal anastomosis and straight coloanal reconstruction. Br J Surg 1995;82:608-10.

7. Hallbook O, Pahlman L, Krog M, Wexner SD, Sjodahl R. Randomized comparison of straight and colonic $\mathrm{J}$ pouch anastomosis after low anterior resection. Ann Surg 1996;224:5865.

8. Rullier E, Laurent C, Bretagnol F, Rullier A, Vendrely V, Zerbib F. Sphincter-saving resection for all rectal carcinomas: the end of the 2-cm distal rule.. Ann Surg. 2005 Mar;241(3):465-9.

9. Rullier E, Sa Cunha A, Couderc P, Rullier A, Gontier R, Saric J. Laparoscopic intersphincteric resection with coloplasty and coloanal anastomosis for mid and low rectal cancer. British Journal of Surgery 2003;90:445-51.

10. Saito N, Moriya Y,et Intersphincteric Resection in Patients with Very Low Rectal Cancer: A Review of the Japanese Experience . Dis Colon Rectum 2006 oct;49(10)s13-s22.
11. Weiser MR, Quah HM, Shia J, Guillem JG, Paty PB, Temple LK, Goodman KA, Minsky BD, Wong WD. Sphincter preservation in low rectal cancer is facilitated by preoperative chemoradiation and intersphincteric dissection. Ann Surg. 2009 Feb;249(2):236-42.

12. Jayne DG, Guillou PJ, Thorpe H, Quirke P, , Smith AM, Heath RM, Brown JM; UK MRC CLASICC Trial Group.Randomized trial of laparoscopic-assisted resection of colorectal carcinoma: 3-year results of the UK MRC CLASICC Trial Group. : J Clin Oncol. $2007 \mathrm{Jul}$ 20;25(21):3061-8.

13. Annamaneni R., Marks J., Curran T., Mohiuddin M., Marks G.. A Comparison of Laparoscopic Vs. Open Radical Sphincter PreservingSurgery for Distal Rectal Cancer Following Neoadjuvant Therapy. Poster Presentation, Philadelphia Academy of Surgery Annual Resident Paper Competition, Philadelphia, Pennsylvania, February 7, 2005.

14. Ramos J.R. Laparoscopic very low anterior resection and coloanal anastomosis using the pull-through technique. Dis Colon Rectum. 1995 Nov; 38(11):1217-

15. Ramos JR.Ressecção Anterior Ultrabaixa e Interesfinctérica com Anastomose Coloanal por Videolaparoscopia: Nota técnica e vídeo. Revista do colégio Brasileiro de Cirurgiões. 2009.

16. Amin AI, Hallbook O, Lee AJ, Sexton R, Moran BJ, Heald RJ. A 5-cm colonic J pouch colo-anal reconstruction following anterior resection for low rectal cancer results in acceptable evacuation and continence in the long term. Colorectal Disease 2003;5(1):33.

17. Dehni N, Tiret E, Singland JD, Cunningham C, Schlegel RD, Guiguet M, Parc R . Long-term functional outcome after low anterior resection: comparison of low colorectal anastomosis and colonic J-pouch-anal anastomosis. Dis Colon Rectum 1998;41:817-226. 
18. Fazio VW, Mantyh CR, Hull TL. Colonic "coloplasty": novel technique to enhance low colorectal or coloanal anastomosis. Dis Colon Rectum 2000; 43:1448-50.

19. Z'Graggen K, Maurer C, Mettler D, Stoupis C, Wildi S, Buchler MW. A novel colon pouch and its comparison with a straight coloanal and colon J-pouch-anal anastomosis: preliminary results in pigs. Surgery 1999;125:105-112.

20. Ho YH, Brown S, Heah SM, Tsang C, Seow-Choen F, Eu KW. Comparison of J-pouch and coloplasty pouch for low rectal cancers: a randomized, controlled trial investigating functional results and comparative anastomotic leak rates. Ann Surg. 2002 jul;236(1):49-55.

21. Huber FT, Herter B, Siewert JR. Colonic pouch vs. side-toend anastomosis in low anterior resection. Dis Colon Rectum, 1999;42(7):896-902.

22. Brown CJ, Fenech DS, McLeod RS. Reconstructive techniques after rectal resection for rectal cancerCochrane Database Syst Rev. 2008 Apr 16;(2):CD006040

23. Hallbook O, J Johansson K, Sjodahl R. Laser Dopller blood flow measurement in the rectal resection for carcinomacomparison between the straight and colonic $\mathrm{J}$ pouch reconstruction. Br J Surg 1996;

24. Gervaz P, Rotholtz N, Wexner SD, You SY, Saigusa N, Kaplan E, Weiss RG, Nogueras JJ, Belin B. Colonic J-pouch function in rectal cancer patients: impact of adjuvant chemoradiotherapy. Dis Colon Rectum 2001;44(11):1667-75.

25. Rullier A, Laurent C, Capdepont M, Vendrely V, Belleannée G, Bioulac-Sage P, Rullier E . Lymph nodes after preoperative chemoradiotherapy for rectal carcinoma: number, status, and impact on survival. Am J Surg Pathol. 2008 Jan;32(1):45-50.

26. Habr-Gama A, Perez RO, Nadalin W, Sabbaga J, Ribeiro U Jr, Silva e Sousa AH Jr, Campos FG, Kiss DR, Gama-Rodrigues J. Operative versus nonoperative treatment for stage 0 distal rectal cancer following chemoradiation therapy: long-term results. Ann Surg. 2004 Oct;240(4):711-7.

27. Portier G, Ghouti L, Kirzin S, Guimbaud R, Rives M, Lazorthes F. Oncological outcome of ultra-low coloanal anastomosis with and without intersphincteric resection for low rectal adenocarcinoma. Br J Surg. 2007 Mar;94(3):341-5.

28. Akasu T, Takawa M, Yamamoto S, Ishiguro S, Yamaguchi T, Fujita S, Moriya Y, Nakanishi Y. Intersphincteric resection for very low rectal adenocarcinoma: univariate and multivariate analyses of risk factors for recurrence. Ann Surg Oncol. 2008 Oct;15(10):2668-76.

29. Ito M, Saito N, Sugito M, Kobayashi A, Nishizawa Y, Tsunoda Y. Analysis of clinical factors associated with anal function after intersphincteric resection for very low rectal cancer. : Dis Colon Rectum. 2009 Jan;52(1):64-70.

30. Tilney HS, Tekkis PP. Extending the horizons of restorative rectal surgery: intersphincteric resection for low rectal cancer Colorectal Dis. 2008 Jan;10(1):3-15.

31. Tsang WW, Chung CC, Kwok SY, Li MK. Laparoscopic sphincter-preserving total mesorectal excision with colonic Jpouch reconstruction: five-year results. Ann Surg. 2006 Mar;243(3):353-8.

32. Laurent C, Leblanc F, Gineste C, Saric J, Rullier E. Laparoscopic approach in surgical treatment of rectal cancer. Br J Surg. 2007 Dec;94(12):1555-61.

\section{Endereço para correspondência:}

JOSÉ REINAN RAMOS

Rua Visconde e Silva, 52 / 701

Botafogo, Rio de Janeiro, RJ

CEP: 22271-090

Tel.: (21) 2286-4996 / 9986-5443

E-mail:j.reinan.ramos@openlink.com.br 